\section{Eventos adversos en la terapia farmacológica de la enfermedad inflamatoria intestinal}

\author{
LITAL MEYER ${ }^{1}$, DANIELA SIMIAN 2 ,a , JAIME LUBASCHER ${ }^{3}$, \\ RAÚL ACUÑA ${ }^{3}$, CAROLINA FIGUEROA ${ }^{3}$, GUILLERMO SILVA ${ }^{3}$, \\ JAVIER BRAHM ${ }^{3}$, RODRIGO QUERA ${ }^{3}$
}

\section{Adverse events associated with the treatment of inflammatory bowel disease}

\begin{abstract}
Background: The purpose of inflammatory bowel disease (IBD) treatment is to achieve resolution of symptoms and remission of disease with a minimum of adverse events (AE). Aim: To report AE of different prescriptions used for the treatment of IBD. Material and Methods: Analysis of a registry of patients with IBD held at a private clinic from 1976 to 2013. All used medications, the occurrence and severity of AE were recorded. Results: The records of $346 \mathrm{pa}$ tients aged 16 to 86 years, $74 \%$ with ulcerative colitis, were analyzed. The most commonly type of medications prescribed were 5-aminosalicylates (5-ASAs) in 329 patients (92\%), followed by adrenal steroids in 218 (61\%). Forty nine AE were recorded in the same number of patents (14\%). These were more common in patients with Crohn disease $(n=19,21 \%)$. An univariate analysis, demonstrated that extra-intestinal manifestations, hospitalizations secondary to IBD crisis, requirement of surgery and treatment with steroids, immunosuppressants or biologic agents were significantly associated with the presence of AE. AEs were more common with immunosuppressants, followed by 5-ASAs and steroids. Discontinuation of therapy was required in 79, 100 and $43 \%$ of patients taking these medications, respectively. Twenty percent of AEs were severe. Leukopenia and pancytopenia along with alopecia were the most common AEs attributable to azathioprine. Conclusions: The occurrence of AEs in patients with IBD is uncommon. Even inmunosuppressants or biologic agents have a low rate of $A E$ and most of them mild.
\end{abstract}

(Rev Med Chile 2015; 143: 7-13)

Key words: Crohn Disease; Colitis, ulcerative; Drug-related side effects and adverse reactions; Inflammatory Bowel Disease.

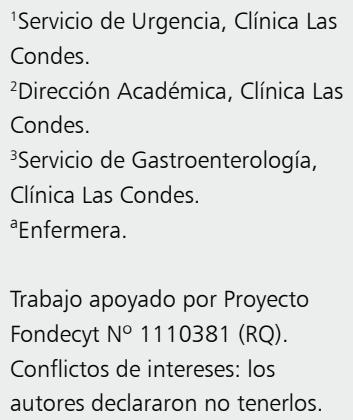

Trabajo apoyado por Proyecto Fondecyt No 1110381 (RQ). Conflictos de intereses: los autores declararon no tenerlos.

Recibido el 2 de septiembre de 2014, aceptado el 1 de diciembre de 2014.

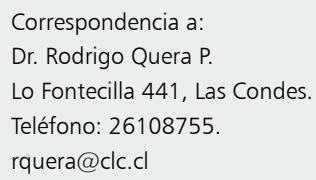

\section{L} a colitis ulcerosa (CU) y la enfermedad de Crohn (EC) son enfermedades inflamatorias intestinales (EII) que surgen de la interacción entre factores genéticos, inmunológicos y ambientales. Su etiología es aún desconocida, por lo que la terapia médica para curarlas aún no está disponible ${ }^{1,2}$. La elección del tratamiento en pacientes con EII depende de varios factores, incluyendo severidad de la enfermedad, localización, fenotipo, presencia de complicaciones y patrón de la enfermedad ${ }^{3,4}$. Este último incluye la frecuencia de recaídas, curso de la enfermedad, presencia de manifestaciones extra-intestinales, respuesta previa a los fármacos y antecedentes de eventos adversos $(\mathrm{EA})^{4,5}$. Es por ello que el tratamiento debe ser individualizado y basado en la respuesta clínica y la tolerancia del paciente a la terapia ${ }^{3}$.

El tratamiento farmacológico actual de los pa- 
cientes con EII comprende el uso de 5-aminosalicilatos (5-ASA), corticoides, inmunomoduladores y terapia biológica ${ }^{6,7}$. Los corticoides son efectivos en inducir la remisión en crisis moderada-severa, sin embargo, posee múltiples y conocidos EA a corto y largo plazo. Debido a su uso generalizado en el tratamiento de la EII, es importante conocer su eficacia y EA asociados ${ }^{7}$. Por otro lado, el uso de inmunomoduladores está bien establecido en el tratamiento de la CU y la EC, probando ser efectivos en la inducción y mantención de la remisión de la enfermedad ${ }^{8}$. Sin embargo, pueden presentarse EA siendo necesaria la disminución de la dosis o la suspensión del fármaco ${ }^{8,9}$.

Aunque la terapia convencional logra controlar la inflamación, esta no cambia el curso de la enfermedad. Más aún, muchos pacientes son refractarios a dichos fármacos. La terapia biológica juega un rol fundamental en el control de los factores proinflamatorios, logrando obtener la remisión de manera rápida y efectiva y su mantención en el tiempo ${ }^{4}$. En la actualidad cuatro fármacos antifactor de necrosis tumoral (anti-TNF $\alpha$ ) han sido aprobados para su uso en EII: infliximab, adalimumab, certolizumab pegol y golimumab. Diferentes estudios han demostrado la eficacia de infliximab tanto en EC como en CU, logrando cumplir los objetivos del tratamiento en estas patologías ${ }^{10}$. Sin embargo, se debe considerar que esta mayor efectividad no está exenta de EA, destacando el riesgo de infecciones y neoplasias ${ }^{4,11-13}$.

Por lo referido, el tratamiento de la EII es un desafío, siendo su principal objetivo lograr la resolución de los síntomas y mantener la remisión con el mínimo de EA en un ambiente seguro y efectivo $^{14}$. En ese sentido, diferentes autores han sugerido la posibilidad de desescalar en la terapia de la EII en aquel grupo que presente un alto riesgo de EA severos o un bajo riesgo de recaída después de suspender el fármaco ${ }^{15}$.

El objetivo de este estudio fue describir los EA de los diferentes esquemas de tratamiento indicados en pacientes con EII.

\section{Material y Métodos}

Estudio descriptivo retrospectivo transversal utilizando un registro de pacientes con EII de Clínica Las Condes, que incluye pacientes diagnosticados entre los años 1976 y 2013. Cada médico gastroenterólogo completó en el registro los datos de los pacientes con diagnóstico confirmado de EII, atendidos entre abril de 2012 y febrero de 2014 en Clínica Las Condes. Tanto el Registro de EII como este estudio en particular, fueron aprobados por el Comité de Ética de la institución.

Se incluyeron datos demográficos y clínicos de la enfermedad: años de enfermedad, edad al diagnóstico, tabaquismo, antecedentes familiares de EII, localización de la enfermedad, patrón de comportamiento de la EC, tratamiento farmacológico, hospitalizaciones por crisis de EII, cirugías relacionadas a EII y EA asociados a cada fármaco.

Se registró el tratamiento utilizado en cada paciente y todos los EA asociados a 5-ASA, corticoides, inmunosupresores (azatioprina (AZA), 6-mercaptopurina (6-MP) y metotrexato) y agentes biológicos (infliximab, adalimumab y natalizumab), clasificándolos por tipo de medicamento. Por otro lado, se agruparon los EA según su severidad, basándose en la escala de severidad de EA del Departamento de Salud de Estados Unidos ${ }^{16}$. Se definió EA leve al evento que requirió suspensión del fármaco y/o uso de otro medicamento para revertir el EA, y EA severo al evento que requirió hospitalización o cirugía para su tratamiento.

En el grupo de pacientes tratados con agentes biológicos, previo al inicio de esta terapia, por protocolo se solicitó prueba de tuberculina (PPD) o quantiferon y radiografía de tórax.

Para el análisis estadístico descriptivo de las variables cualitativas y cuantitativas se utilizó el software R Commander. Las variables numéricas continuas se analizaron con mediana e intervalos ya que no mostraban distribución normal y se compararon mediante test de Mann Whitney. Para las variables cualitativas categóricas se utilizó frecuencia relativa porcentual y para el análisis estadístico comparativo de ellas se utilizó chi cuadrado. Se determinó significación estadística a un valor $\mathrm{p}<0,05$.

\section{Resultados}

Durante el período estudiado se incluyeron 364 pacientes con EII, 74\% de ellos con CU. La mediana de edad fue 36 años (intervalo: 16-86). La mediana de años de enfermedad fue de 6 años (intervalo: 1-38). En relación al tratamiento, 
los 5-ASA fueron los fármacos más utilizados $(\mathrm{n}=329,92 \%)$, seguidos por los corticoides $(\mathrm{n}=218,61 \%)$. Han recibido terapia biológica 41 pacientes (39 con infliximab, 2 con adalimumab y 1 con natalizumab). Las características demográficas y clínicas se muestran en la Tabla 1.

Se registraron 49 EA (14\%), correspondiendo cada evento a un paciente distinto. En relación al

\section{Tabla 1. Caracterización demográfica y clínica de pacientes con enfermedad inflamatoria intestinal (EII) por patología}

\begin{tabular}{|c|c|c|}
\hline & $\begin{array}{c}\text { CU } \\
n=264 \\
(\%)\end{array}$ & $\begin{array}{c}\text { EC } \\
\mathrm{n}=92 \\
(\%)\end{array}$ \\
\hline Género femenino, n (\%) & $147(55,7)$ & $49(33,3)$ \\
\hline $\begin{array}{l}\text { Edad al diagnóstico } \\
\leq 16 \text { años } \\
17-40 \text { años } \\
>40 \text { años }\end{array}$ & $\begin{array}{r}9(3,4) \\
204(77,3) \\
51(19,3)\end{array}$ & $\begin{array}{l}4(4,3) \\
58(63) \\
30(32,6)\end{array}$ \\
\hline $\begin{array}{l}\text { Hábito tabáquico } \\
\text { Fumador activo } \\
\text { Ex fumador }\end{array}$ & $\begin{array}{l}27(10,2) \\
27(10,2)\end{array}$ & $\begin{array}{l}12(13) \\
11(12)\end{array}$ \\
\hline Antecedente familiar de Ell & $25(9,5)$ & $8 \quad(8,7)$ \\
\hline $\begin{array}{l}\text { Localización enfermedad en CUa } \\
\text { Extensa } \\
\text { Izquierda } \\
\text { Proctitis }\end{array}$ & $\begin{array}{r}136(51,5) \\
48(18,2) \\
80(30,3)\end{array}$ & \\
\hline $\begin{array}{l}\text { Localización enfermedad ECa } \\
\text { Gl superior } \\
\text { Íleon terminal } \\
\text { Ileocolónica } \\
\text { Colon }\end{array}$ & & $\begin{array}{l}3(3,3) \\
31(33,7) \\
26(28,3) \\
34(37)\end{array}$ \\
\hline $\begin{array}{l}\text { Patrón de comportamiento ECa } \\
\text { Inflamatoria } \\
\text { Estenosante } \\
\text { Fistulizante } \\
\text { Perianal }\end{array}$ & & $\begin{array}{r}74(80,4) \\
10(10,9) \\
7 \quad(7,6) \\
28(30,4)\end{array}$ \\
\hline $\begin{array}{l}\text { Tratamiento farmacológico } \\
\text { 5-Aminosalicilatos } \\
\text { Corticoides } \\
\text { Inmunosupresores } \\
\text { Agentes biológicos }\end{array}$ & $\begin{array}{r}261(98,9) \\
158(59,8) \\
85(32,2) \\
13 \quad(4,9)\end{array}$ & $\begin{array}{l}68(73,9) \\
60(65,2) \\
61(66,3) \\
28(30,4)\end{array}$ \\
\hline Hospitalizaciones por crisis Ell & $84(31,8)$ & $49(53,3)$ \\
\hline Cirugías relacionadas a Ell & $19(7,2)$ & $41(44,6)$ \\
\hline Evento adverso a medicamento & $31(11,7)$ & $19(20,7)$ \\
\hline
\end{tabular}

aEn relación a Clasificación de Montreal. tipo de EII, los pacientes con EC presentaron más EA, al compararlos con el grupo con CU (20,7\% vs $11,7 \%, p=0,01)$. Los EA se presentaron con mayor frecuencia en pacientes entre los 17 y 40 años, siendo este grupo etario el predominante en la población estudiada.

En el análisis univariado de los factores asociados a la presencia de EA en pacientes con EII, las manifestaciones extraintestinales, las hospitalizaciones por crisis de EII, la presencia de cirugías relacionadas a EII, la localización de la enfermedad en CU y el tratamiento con corticoides, inmunosupresores y agentes biológicos tuvieron una relación estadísticamente significativa (Tabla 2).

Del total de EA, los más frecuentes fueron en relación al uso de inmunosupresores, seguido por 5-ASA y corticoides (Figura 1).

La suspensión de la terapia farmacológica en pacientes que presentaron EA fue de $100 \%$ con 5-ASA. Este porcentaje disminuyó a 79\% en pacientes con inmunosupresores y $67 \%$ en los con terapia biológica.

Al considerar todos los pacientes que presentaron un EA, 20\% correspondió a EA categorizados como severos y de este subgrupo, $70 \%$ fue secundario a los inmunosupresores. No se encontraron diferencias significativas al comparar la severidad de los EA por medicamento.

$\mathrm{Al}$ analizar los EA por fármaco, leucopenia y alopecia fueron los EA que se asociaron con mayor frecuencia al uso de AZA (Tabla 3). En el grupo con terapia biológica, los EA se presentaron en 3 casos (todos habían sido tratados con infliximab): herpes zoster, parestesia de extremidades y shock anafiláctico.

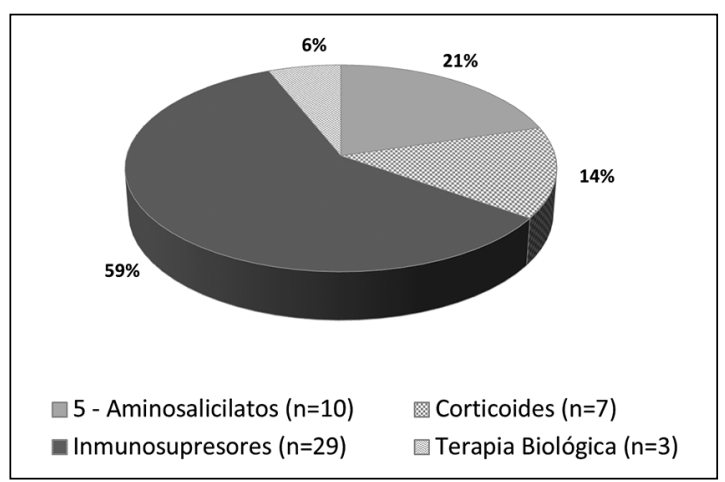

Figura 1. Eventos Adversos $(n=49)$. Porcentaje por tipo de fármaco. 
Tabla 2. Análisis univariado de factores asociados a la presencia de eventos adversos a medicamentos en pacientes con enfermedad inflamatoria intestinal (EII)

\begin{tabular}{|c|c|c|c|}
\hline & $\begin{array}{c}\text { EA } \\
n=49(\%)\end{array}$ & $\begin{array}{c}\text { No EA } \\
n=315(\%)\end{array}$ & Valor $\mathbf{p}$ \\
\hline \multicolumn{4}{|l|}{ Patología } \\
\hline Colitis ulcerosa & $29(59,2)$ & $235(74,6)$ & \multirow{3}{*}{$0,019^{a}$} \\
\hline Enfermedad de Crohn & $19(38,8)$ & $73(23,2)$ & \\
\hline Ell no clasificable & $1(2)$ & $7 \quad(2,2)$ & \\
\hline Sexo femenino & $31(63,23)$ & $172(54,6)$ & 0,256 \\
\hline \multicolumn{4}{|l|}{ Edad al diagnóstico } \\
\hline$\leq 16$ años & $2(4,1)$ & $11(3,5)$ & \multirow{3}{*}{0,650} \\
\hline 17-40 años & $33(67,3)$ & $232(73,7)$ & \\
\hline$>40$ años & $14(28,6)$ & $72(22,9)$ & \\
\hline \multicolumn{4}{|l|}{ Hábito tabáquico } \\
\hline Fumador activo & $2(4,1)$ & $38(12,1)$ & 0,096 \\
\hline Ex fumador & $5(10,2)$ & $33(10,5)$ & 0,953 \\
\hline Antecedente familiar de Ell & $7(14,3)$ & $26(8,3)$ & 0,171 \\
\hline Manifestaciones extraintestinales & $30(61,2)$ & $117(37,1)$ & 0,001 \\
\hline \multicolumn{4}{|l|}{ Localización enfermedad en CUb } \\
\hline Extensa & $22(75,9)$ & $114(48,5)$ & \multirow{3}{*}{0,008} \\
\hline Izquierda & $5(17,2)$ & $43(18,3)$ & \\
\hline Proctitis & $2(6,9)$ & $78(33,2)$ & \\
\hline \multicolumn{4}{|l|}{ Localización enfermedad en $\mathrm{EC}^{\mathrm{b}}$} \\
\hline Gl superior & $1(5,3)$ & $2(2,7)$ & \multirow{4}{*}{0,029} \\
\hline íleon terminal & $6(31,6)$ & $24(34,2)$ & \\
\hline Ileocolónica & $5(26,3)$ & $21(28,8)$ & \\
\hline Colon & $8(42,1)$ & $26(35,6)$ & \\
\hline \multicolumn{4}{|l|}{ Patrón de comportamiento $\mathrm{EC}^{\mathrm{b}}$} \\
\hline Inflamatoria & $13(68,4)$ & $61(83,6)$ & \multirow{3}{*}{0,228} \\
\hline Estenosante & $4(21,1)$ & $6(8,2)$ & \\
\hline Fistulizante & $2(10,5)$ & $5(6,8)$ & \\
\hline Compromiso perianal & $8(42,1)$ & $20(27,4)$ & 0,214 \\
\hline \multicolumn{4}{|l|}{ Tratamiento farmacológico } \\
\hline 5-Aminosalicilatos & $43(87,8)$ & $294(93,3)$ & 0,165 \\
\hline Corticoides & $42(85,7)$ & $180(57,1)$ & 0,0001 \\
\hline Inmunosupresores & $42(85,7)$ & $108(34,3)$ & $<0,0001$ \\
\hline Agentes biológicos & $13(26,5)$ & $29 \quad(9,2)$ & 0,0004 \\
\hline Hospitalizaciones por crisis Ell & $32(65,3)$ & $103(32,7)$ & $<0,0001$ \\
\hline Cirugías relacionadas a Ell & $17(34,7)$ & $43(13,7)$ & 0,0002 \\
\hline
\end{tabular}

${ }^{a} \mathrm{Al}$ comparar EC y CU. ${ }^{b}$ En relación a Clasificación de Montreal.

\section{Discusión}

En la EII se busca que el tratamiento logre la mayor eficacia en su remisión, idealmente con curación de la mucosa. En segundo lugar, lograr esto con la mayor seguridad y mínima toxicidad ${ }^{17}$. A nuestro conocimiento, esta sería la primera publicación que describe la presencia de EA secundarios a fármacos en una población chilena con EII. En nuestro estudio, uno de cada seis pacientes presentó un EA, con mayor frecuencia en pacientes con EC, coincidiendo con lo descrito en la literatura ${ }^{3}$.

En esta serie, 21\% de los EA se presentó en rela- 
Tabla 3. Eventos adversos asociados al uso de inmunosupresores en enfermedad inflamatoria intestinal (EII)

\begin{tabular}{|lc|}
\hline Evento Adverso AZA & $\mathbf{n}=\mathbf{1 4 6}(\mathbf{\%})$ \\
Leucopenia & $11(7,5)$ \\
Alopecia & $5(3,4)$ \\
Pancreatitis & $3(2)$ \\
Hepatitis/Alt. pruebas hepáticas & $2(1,4)$ \\
Herpes Zoster & $2(1,4)$ \\
Rush Urticarial & $2(1,4)$ \\
Intolerancia & $3(2)$ \\
Otro & $1(0,7)$ \\
\hline
\end{tabular}

ción al uso de 5-ASA, porcentaje que se encuentra dentro de lo señalado en la literatura, a pesar de que el rango es bastante amplio (13 a 73\% $)^{18,19}$. Loftus et al, en una revisión sistemática, señalaron que sólo 0 a $9 \%$ de los pacientes que presentaron EA a este fármaco debieron suspenderlo, lo cual difiere ampliamente en nuestra población, donde $100 \%$ de los pacientes suspendió esta terapia. Si bien 5-ASA no es un fármaco inmunosupresor, su uso no está exento de EA severos. La mayoría de las publicaciones de EA con 5-ASA se basan en casos o series de casos clínicos, siendo el daño renal y la pancreatitis los descritos con mayor frecuencia y ambos fenómenos idiosincráticos ${ }^{20}$.

En relación al uso de corticoides, está demostrada su eficacia en la EII, pero se relaciona a EA significativos a corto plazo como efectos cosméticos (acné, cara de luna, estrías en la piel) o intolerancia a la glucosa y a largo plazo como cataratas, osteoporosis, osteonecrosis, miopatía y riesgo de infecciones oportunistas. Hay estudios señalando que el riesgo de infección es mayor al compararlos con tiopurínicos o terapia biológi$\mathrm{ca}^{13}$. Estos antecedentes deben ser considerados al momento de evaluar pacientes que evolucionan con una corticodependencia. En nuestro estudio, al considerar todos los pacientes que presentaron EA, $14 \%$ estuvo relacionado con el uso de corticoides, siendo necesario suspenderlos en $43 \%$ de los pacientes. Debido al riesgo de EA asociados a los corticoides sistémicos clásicos, existe la posibilidad de utilizar budesonida en pacientes con EC con compromiso inflamatorio de íleon terminal y colon ascendente, con una efectividad algo menor al compararlo con prednisona, pero con menor riesgo de $\mathrm{EA}^{21,22}$. Estudios recientes han demostrado la efectividad de budesonida de liberación prolongada en $\mathrm{CU}$, siendo una opción en aquellos pacientes que no responden a 5-ASA en dosis adecuadas, evitando con ello el riesgo de los EA descritos con prednisona ${ }^{5,14}$. Dado que nuestro estudio es retrospectivo, no fue posible definir si aquellos pacientes con EC tratados con budesonida presentaron un menor porcentaje de EA comparados con pacientes tratados con prednisona.

El uso de tiopurinas (AZA/6-MP) está establecido en los algoritmos de tratamiento de las EII, ya sea EC o CU. Estos han demostrado ser efectivos en mantener la remisión ${ }^{4,5,8}$, consiguiendo incluso la curación de la mucosa en $37 \%$ y $16,5 \%$ de los pacientes con CU y EC, respectivamente ${ }^{17}$. La mayor limitación del uso de AZA/6-MP son los EA a corto y largo plazo, que ocurren en alrededor de 9 a $32 \%$ de los pacientes y que requieren reducción de la dosis o suspensión de la terapia, en 10\% de ellos, aproximadamente ${ }^{3,8,23}$. En nuestro estudio, del total de pacientes con inmunosupresores, $20 \%$ presentó EA, cifras similares a las descritas en la literatura. Es importante mencionar que en esta muestra, los EA motivaron la suspensión del tratamiento en $79 \%$, más cercano a lo descrito en el estudio de Lopez-Martin et al, donde $84 \%$ de los pacientes que presentó EA por AZA $(13,5 \%)$ suspendieron la terapia ${ }^{3}$. Los EA más comunes en relación al uso de AZA fueron la supresión de la médula ósea, presentado en 7,5\% de los pacientes, un poco mayor a 2 a $5 \%$ señalado en la literatura, seguido por alopecia $(3,4 \%)$ y pancreatitis $(2 \%)$. La hepatotoxicidad fue infrecuente, presentándose sólo en $1,4 \%$ de los pacientes, siendo este porcentaje algo menor a lo descrito en la literatura ( 2 a $4 \%)^{3,9,14,17,23}$. Se ha descrito que los pacientes tratados con tiopurinas tienen tres veces más riesgo de retirarse de estudios de investigación debido a los EA presentados (alergia, leucopenia, pancreatitis y náuseas/vómitos) al compararlo con el grupo tratado con placebo ${ }^{14}$. Pacientes que presentan EA a AZT leves (intolerancia oral con náuseas, vómitos o dolor abdominal) pueden ser tratados con 6-MP con buenos resultados en relación a tolerancia ${ }^{24}$. En nuestro estudio, por ser retrospectivo, esta variable no pudo ser medida. Por otra parte, se ha sugerido que la medición 
de los metabolitos de las tiopurinas (nucleótidos tioguaninas y metilmercaptopurina) y de la actividad de la tiopurinometiltransferasa permitiría evaluar la dosis de estos fármacos optimizando su efectividad y seguridad ${ }^{22}$.

El uso indefinido de AZA se asocia con mayor riesgo de desarrollar linfoma, con un riesgo relativo de $4,18^{14}$. Sin embargo, se ha planteado que la EII en sí misma podría estar asociada a un mayor riesgo de enfermedad linfoproliferativa ${ }^{17}$. Pacientes tratados con tiopurínicos (AZT/6-MP) por más de un año presentan un mayor riesgo de cáncer de piel no-melanoma, el cual se mantiene incluso después de haber suspendido estos fármacos ${ }^{17,25}$. Pacientes que inician tratamientos con AZT/6-MP deben evitar la exposición a rayos ultravioleta para disminuir el riesgo de cáncer de piel y deben tener un control anual con el dermatólogo ${ }^{25}$. En nuestro estudio, ningún paciente tratado con AZT/6-MP ha desarrollado alguna neoplasia asociada al uso de estos fármacos, sin embargo, estos resultados deben ser evaluados con cautela dado el corto período de tiempo de tratamiento con esta terapia.

El desarrollo de terapias biológicas ha sido de gran impacto para los pacientes con EII, demostrando su eficacia en lograr remisión libre de esteroides, curación de la mucosa y cambiar la historia natural de la EII ${ }^{10,26}$. En nuestro estudio, los pacientes con EII tratados con terapia biológica presentaron la menor frecuencia de EA: sólo 6\% del total de EA. Las infecciones son la mayor preocupación cuando se utiliza agentes biológicos; sin embargo, estas son menores al compararlas con el uso de esteroides ${ }^{5,13}$. Se han descrito algunos casos de tuberculosis asociada a infliximab en pacientes con EII. Por esta razón, antes de iniciar cualquier terapia biológica se debe evaluar a los pacientes con test de tuberculina (PPD) y radiografía de tórax, e iniciar previamente tratamiento para tuberculosis en caso de ser necesario ${ }^{12-14}$. Pacientes que ya se encuentren en tratamiento inmunosupresor (AZT, 6-MP o metotrexato), deben ser evaluados con quantiferon para disminuir el riesgo de falsos negativos con el $\mathrm{PPD}^{12}$. En nuestro estudio una paciente con quantiferon positivo requirió uso de isoniazida como profilaxis en conjunto con el inicio de terapia biológica ${ }^{13}$, evolucionado sin evidencias de tuberculosis. Para disminuir el riesgo de infección por uso de inmunosupresores o terapia biológica, es necesario, previo al inicio de estos fármacos, una evaluación de la presencia de infección por virus hepatitis A (VHA), virus hepatitis $\mathrm{B}(\mathrm{VHB})$ y virus de inmunodeficiencia humana $(\mathrm{VIH})$, indicando las vacunas respectivas (VHA, VHB, pneumococo, influenza, etc.) según normas publicadas ${ }^{13,27}$.

La principal limitación de nuestro estudio es que al ser retrospectivo transversal, existe la posibilidad de sesgo de memoria en los pacientes y el equipo tratante en relación a la presencia de EA asociados a algún fármaco $y$, además, no permite el seguimiento de las medidas adoptadas en relación a cada EA.

En conclusión, nuestro estudio señala que la presencia de EA en una muestra de pacientes con EII en tratamiento es infrecuente, incluso en aquellos tratados con inmunosupresores y terapia biológica. Pese a ello, es fundamental una monitorización cercana de los pacientes con EII, con el fin de definir caso a caso cuál es la mejor opción terapéutica, considerando los beneficios y riesgos de cada una de ellas. El temor a la presencia de EA no debe ser la causa para diferir una estrategia terapéutica cuando la condición del paciente así lo amerita.

\section{Referencias}

1. Dignass A, Eliakim R, Magro F, Maaser C, Chowers Y, Geboes K, et al. Second European evidence-based consensus on the diagnosis and management of ulcerative colitis part 1: definitions and diagnosis. J Crohns Colitis 2012; 6: 965-90.

2. Van Assche G, Dignass A, Panes J, Beaugerie L, Karagiannis J, Allez M, et al. The second European evidencebased Consensus on the diagnosis and management of Crohn's disease: Definitions and diagnosis. J Crohns Colitis 2010; 4: 7-27.

3. López-Martín C, Chaparro M, Espinosa L, Bejerano A, Maté J, Gisbert JP. Adverse events of thiopurine immunomodulators in patients with inflammatory bowel disease. Gastroenterol Hepatol 2011; 34: 385-92.

4. Dignass A, Lindsay JO, Sturm A, Windsor A, Colombel JF, Allez M, et al. Second European evidence-based consensus on the diagnosis and management of ulcerative colitis part 2: current management. J Crohns Colitis 2012; 6: 991-1030.

5. Dignass A, Van Assche G, Lindsay JO, Lémann M, Söderholm J, Colombel JF, et al. The second European evidence-based Consensus on the diagnosis and management of Crohn's disease: Current management. J Crohns Colitis 2010; 4: 28-62. 
6. Ford AC, Achkar JP, Khan KJ, Kane SV, Talley NJ, Marshall JK, et al. Efficacy of 5-aminosalicylates in ulcerative colitis: systematic review and meta-analysis. Am J Gastroenterol 2011; 106: 601-16.

7. Ford AC, Bernstein CN, Khan KJ, Abreu MT, Marshall JK, Talley NJ, et al. Glucocorticosteroid therapy in inflammatory bowel disease: systematic review and metaanalysis. Am J Gastroenterol 2011; 106: 590-9.

8. Hindorf U, Lindqvist M, Hildebrand H, Fagerberg U, Almer S. Adverse events leading to modification of therapy in a large cohort of patients with inflammatory bowel disease. Aliment Pharmacol Ther 2006; 24: 331-42.

9. Nielsen $\mathrm{OH}$, Bjerrum JT, Herfarth H, Rogler G. Recent advances using immunomodulators for inflammatory bowel disease. J Clin Pharmacol 2013; 53: 575-88.

10. Simian D, Quijada MI, Lubascher J, Acuña R, Quera R. Rol de infliximab en enfermedad inflamatoria intestinal: Experiencia local. Rev Med Chile 2013; 141: 1158-65.

11. Ford AC, Peyrin-Biroulet L. Opportunistic Infections With Anti-Tumor Necrosis Factor-Therapy in Inflammatory Bowel Disease: Meta-Analysis of Randomized Controlled Trials. Am J Gastroenterol 2013; 108: 1268-76.

12. Targownik LE, Bernstein CN. Infectious and Malignant Complications of TNF Inhibitor Therapy in IBD. Am J Gastroenterol 2013; 108: 1835-42.

13. Rahier JF, Magro F, Abreu C, Armuzzi A, Ben-Horin S, Chowers Y, et al. Second European evidence-based consensus on the prevention, diagnosis and management of opportunistic infections in inflammatory bowel disease. J Crohns Colitis 2014; 8: 443-68.

14. Blonski W, Buchner AM, Lichtenstein GR. Inflammatory Bowel Disease Therapy. Curr Opin Gastroenterol 2011; 27: 346-57.

15. Pariente B, Laharie D. Review article: why, when and how to de-escalate therapy in inflammatory bowel diseases. Aliment Pharmacol Ther 2014; 40: 338-53.

16. National Cancer Institute. Common Terminology Criteria for Adverse Events v4.0. NCI, NIH, DHHS. May 29, 2009. NIH publication \# 09-7473. Disponible en:http:// evs.nci.nih.gov/ftp1/CTCAE/CTCAE_4.03_2010-06-14_ QuickReference_5x7.pdf. [Consultado en 2 de julio de 2014].
17. Bär F, Sina C, Fellermann K. Thiopurines in inflammatory bowel disease revisited. World J Gastroenterol 2013; 19: 1699-706.

18. Loftus EV Jr, Kane SV, Bjorkman D. Systematic review: short-term adverse effects of 5-aminosalicylic acid agents in the treatment of ulcerative colitis. Aliment Pharmacol Ther 2004; 19: 179-89.

19. Ford AC, Kane SV, Khan KJ, Achkar JP, Talley NJ, Marshall JK, et al. Efficacy of 5-aminosalicylates in Crohn's disease: systematic review and meta-analysis. Am J Gastroenterol 2011; 106: 617-29.

20. Van Bodegraven AA, Mulder CJ. Indications for 5-aminosalicylate in inflammatory bowel disease: is the body of evidence complete? World J Gastroenterol 2006; 12: 6115-23.

21. Danese S, Siegel CA, Peyrin-Biroulet L. Review article: integrating budesonide-MMX into treatment algorithms for mild-to-moderate ulcerative colitis. Aliment Pharmacol Ther 2014; 39: 1095-103.

22. Irving PM. Can we get more from our current treatments? Best Pract Res Clin Gastroenterol 2014; 28: 451-63.

23. Costantino G, Furfaro F, Belvedere A, Alibrandi A, Fries W. Thiopurine treatment in inflammatory bowel disease: response predictors, safety, and withdrawal in follow-up. J Crohns Colitis 2012; 6: 588-96.

24. Kennedy NA, Rhatigan E, Arnott ID, Noble CL, Shand AG, Satsangi J, et al. A trial of mercaptopurine is a safe strategy in patients with inflammatory bowel disease intolerant to azathioprine: an observational study, systematic review and meta-analysis. Aliment Pharmacol Ther 2013; 38: 1255-66.

25. Ariyaratnam J, Subramanian V. Association Between Thiopurine Use and Nonmelanoma Skin Cancers in Patients With Inflammatory Bowel Disease: A MetaAnalysis. Am J Gastroenterol 2014; 109: 163-9.

26. De Vroey B, Colombel JF. IBD in 2010: optimizing treatment and minimizing adverse events. Nat Rev Gastroenterol Hepatol 2011; 8: 74-6.

27. Magro F, Abreu C. Immunisations in Crohn's disease: who? why? what? when? Best Pract Res Clin Gastroenterol 2014; 28: 485-96. 